\section{Thalassaemia prior and conse- quent to COVID-19 pandemic. The perspective of Thalassaemia International Federation (TIF)}

\author{
Androulla Eleftheriou, Lily Cannon, \\ Michael Angastiniotis
}

Thalassaemia International Federation, Nicosia, Cyprus

\begin{abstract}
Patients with haemoglobin disorders, particularly $\beta$-thalassaemia or sickle cell disease (SCD) or combined forms, on account of their underlying disease pathology and associated (iron load mainly in the case of thalassaemia) co-morbidities are defined as high-risk individuals prone to develop more severe complications from coronavirus disease-2019 (COVID-19). Despite the fact that epidemiological evidence concerning severe acute respiratory syndrome-coronavirus-2 (SARS-CoV-2) infection in these patients is currently limited across the world, it is expected that COVID-19 pandemic will have a very serious, negative impact on national economies, healthcare and social systems and consequently significant respective repercussions on the patients particularly chronic ones, and their families. Although this may be a temporary challenge in some countries of high HDI and robust health, public health and social infrastructures, this can be a long term challenge with serious to tragic consequences in countries particularly devoid of universally covered heath care systems.
\end{abstract}

Thalassaemia International Federation (TIF) in this present paper summarises the key challenges as expressed by the patients, their families and involved health care professionals themselves prior and consequent to COVID-19 pandemic, describes its response during the pandemic and expresses its position in support of its global patient community.

\section{Introduction}

Thalassaemia and sickle cell disease (SCD) are the most common monogenic disorders in human. About $7 \%$ of the global population carry an abnormal haemoglobin gene. ${ }^{1}$ These disorders are characterised by multi-systemic involvement and need for intensive lifelong therapy and multidisci- plinary care., ${ }^{2,3}$ If left ineffectively controlled and managed, these disorders are associated with high rates of morbidity, poor quality of life and early death.

On the other hand and consequent to the dramatic scientific progress achieved particularly in the last 2-3 decades in the area of the control of these disorders, one may today safely state that these disorders can be effectively prevented and well managed when national disease specific strategies and programmes are established and appropriately monitored for their effectiveness and outcomes.

Survival rates, age distribution and reduction of morbidity and mortality rates constitute important indicators of the success and effectiveness of such programmes and these are seen particularly in the Western world where, however less than $20 \%$ of the global patient population is born and lives. In most countries of the Western world, patients with haemoglobin disorders, particularly those born after the 1980s, have been growing normally, have achieved a very satisfactory quality of life and almost full social integration. ${ }^{1}$ In these settings, patients are now facing a new spectrum of co-morbidities related to ageing. ${ }^{4}$

The challenges however are multiple and magnified for more than $80 \%$ of our patients, living in developing and lowincome countries, This is happening mainly because different priorities exist in the area of public health, including communicable diseases, different priorities in non-communicable diseases but also on account of prevailing poor resources and weak health infrastructures. These, in conjunction with the absence of universal-coverage health care systems in the greatest part of the developing world result in the provision of suboptimal medical and other care to patients particularly those with chronic disorders including patients with haemoglobin disorders. Under such conditions, the risk of these patients developing serious multiple medical complications, including cardiac, hepatic, endocrine disease and other, is very high.

In this widely heterogeneous state of affairs across the globe which reflects the widely different quality in the standards of medical and other care provided to our patients within and across countries, within and across the different regions of the word, the coronavirus disease-2019 (COVID-19) pandemic made in the early 2020 its unexpected grand entry.

Through the findings of the study presented in this report, Thalassaemia International Federation (TIF) aims to proceed with a global appeal to the Governments and to the official internation-
Correspondence: Androulla Eleftheriou, Virologist, Executive Director, Thalassaemia International Federation; 31, Ifigenias Street, 2007 Strovolos, Nicosia, Cyprus.

Tel.: +35722319129.

E-mail: thalassaemia@cytanet.com.cy

Key words: Thalassaemia; sickle cell disease; haemoglobinopathies; COVID-19; SARSCoV-2; coronavirus; pandemic.

Received for publication: 26 May 2020.

Revision received: 29 June 2020.

Accepted for publication: 29 June 2020

This work is licensed under a Creative Commons Attribution 4.0 License (by-nc 4.0).

(Copyright: the Author(s), 2020

Licensee PAGEPress, Italy

Thalassemia Reports 2020; 10:9138

doi:10.4081/thal.2020.9138

al health bodies for their careful consideration and prompt action with regards to COVID-19 and its impact on haemoglobin disorders. This is an effort to prevent serious drawbacks in the health and quality of life of patients with these disorders particularly in the developing world where more than $80 \%$ are born and live.

\section{Methodology}

This report is based on: i) a survey that was conducted prior to COVID-19 pandemic between January - December 2018 with the contribution of 62 National Thalassaemia Associations, in 48 countries representing 3,500 patients globally and on; ii) a second survey that was conducted during the COVID-19 pandemic, between March $15^{\text {th }}$ - May $15^{\text {th }} 2020$ with the contribution of 48 National Thalassaemia Associations, in 42 countries representing 3,200 patients globally.

This report is focused on COVID-19 and its medical, public health, social and economic impact on haemoglobin disorders and compares the pre COVID-19 prevailing environment with regards to haemoglobin disorders across with the post COVID-19 period. TIF has utilised many and multiple communication tools to gain a comprehensive and representative patients' and health care professionals' view, at the global level, on the effects this pandemic has had on the health, medical and other care and on the daily lives of patients and their families.

As an umbrella organisation, safeguarding the rights of patients across the six regions of the world as defined by the WHO 
[African Region (AFRO), Region of the Americas (PAHO), South-East Asia Region (SEARO), European Region (EURO), Eastern Mediterranean Region (EMRO), Western Pacific Region (WPRO)] TIF keeps, with the support of its members, medical/scientific and other collaborators and importantly published literature, reliable information on the status of control (i.e prevention and management) policies existing in 64 countries - members of TIF across the world. Regular contact with its members is maintained through: i) its Board Members (TIF Board of Directors: $14 \mathrm{mem}$ bers (country specific) and 2 Honorary members); ii) national associations (232 from 64 countries); iii) more recently through its newly developed global patient Community Advisory Board including members from 34 countries; and iv) through its ( 350 currently) panel of medical and scientific advisors at national, regional and international level. Communication is achieved mainly through regular electronic contact, the social media and platforms such as the TPC (Thalassaemia Patient Connect Platform) and well-structured topic specific surveys

(e.g. (https://www.surveymonkey.com/r/JY7SGJ T)). More recently and in the context of the pandemic, these tools have been strengthened and complemented through the use of virtual meetings in the context of webinars. TIF participated/contributed in a number of webinars organised by: i) TIF with members of its patient community advisory board and medical advisory network; ii) TIF in collaboration with official regulatory or other professional health oriented bodies (e.g. EHA, ISBT, EMA, WHO); iii) Others including Medical Associations (mainly in the field of haematology and paediatric), the patient community, other collaborators at country level and the industry.

Twenty (20) such virtual meetings were completed at the time this report was prepared (May 20 $0^{\text {th }} 2020$ ) with the participation of 26 member associations, 150 patients and 26 healthcare professionals from 22 countries across Europe, Middle East, South East Asia and West Pacific.

TIF is particularly interested in closely monitoring how and to what extent as compared to pre COVID-19 situation, the 'newly' developed health and social environments consequent to COVID-19 pandemic, at country, regional and international level have affected its patients and how they will impact in the near and long-term future on their health and lives.

\section{TIF's response to COVID-19 pan- demic}

The response of TIF to the needs expressed through the communication channels since early March 2020 includes, and certainly not limited to, the development by patients and health care professionals members of TIF's Advisory Panel, translation and distribution (electronic and hard copies) of a series of informational leaflets on COVID-19-related matters such as:

\section{$\checkmark$ The COVID-19 Pandemic \&} Haemoglobin Disorders

$\checkmark$ A Useful Health \& Nutrition Short Guide for the COVID-19 Pandemic

$\checkmark$ A TIF-Proposed Haemoglobinopathy Patient Pathway during the COVID-19 Pandemic

$\checkmark$ Q\&A: The COVID-19 Pandemic \& the Haemoglobinopathy Patient

$\checkmark$ TIF Position Statement: The inclusion of thalassaemia and sickle cell disease patients in the High Risk Populations

$\checkmark$ Developing Vaccinations \& Therapeutic Drugs for COVID-19

$\checkmark$ Blood \& COVID-19 Guide

$\checkmark$ COVID-19 Pandemic \& Haemoglobin Disorders: A Classification of Risk Groups \& Other Considerations

$\checkmark$ COVID-19 - Thalassaemia \& Sickle Cell Disease: Classification of Risk Groups \& Other Considerations

The above material, is uploaded on TIF's website (https://thalassaemia.org.cy/ tif-publications/), promoted through its social media and disseminated through electronic communication to patients, health care professionals, decision making bodies in more than 60 country members of TIF globally. The material is updated on a weekly basis.

As more information and data continues to emerge, TIF remains committed to continuously update this material and furthermore to continue interacting, better understanding and observing the true patients' perspective in the widely heterogeneous conditions with regards to the prevention, medical and other care of haemoglobin disorders before and during COVID-19 pandemic across the world

\section{Results}

\section{Prior to COVID-19 pandemic}

More than $80 \%$ of the patients with haemoglobin disorders are born and live in the developing countries of the world.

Prior to COVID-19 pandemic and based on the information collected as described in the Methodology, the following findings reflect the then and prior to the COVID-19 pandemic, prevailing situation: i) A significant percentage of patients $(64.8 \%)$ with haemoglobin disorders, mainly but not restricted to, patients with transfusion dependent thalassaemia, do not have access or have interrupted access to adequate blood necessary to keep their haemoglobin to the levels recommended by international standards of care. ${ }^{2}$ The development thus of related medical complications of varying severity affecting the survival and quality of life of these patients is inevitable; ii) A large proportion of patients $(62.8 \%)$ is still receiving, inappropriately or sub-optimally, for the needs of haemoglobin disorders, processed blood (RBCs); iii) Only less than $10 \%$ of the patients $(8.4 \%)$ has access or uninterrupted access to appropriate, for their needs, iron chelation treatment; iv) Less than $5 \%$ of the patients $(4.8 \%)$ are provided with regular well-structured iron load monitoring and multidisciplinary care by experts in the field across different medical disciplines; v) The absence of universal coverage in the context of the healthcare systems in these countries adds significantly to the confinement of patients' access to the necessary, for their disease, services. The financial impact on the patients and their families is immense as a big percentage of patients $(72.5 \%)$ have to pay for the cost of various health services even essential basic ones (including blood in some countries) leading the patients and their families to financially catastrophic situations.

\section{Consequent to COVID-19 pandemic (information and data received dur- ing the pandemic)}

The information below provided by patients' and health professionals' responses to TIF's surveys, as described in Methodology, reflects to a great extend reliably the environment developed consequent or related to the pandemic.

COVID-19 pandemic created new challenges both in the Western world but very sadly magnified existing, already many and multiple challenges particularly in the developing world

TIF has recorded the following challenges in order of priority as expressed by the patients and on many occasions by the involved health care professionals themselves:

Moderate to severe blood shortages leading to moderate to severe drops of the pre-transfusion haemoglobin levels. In the developing countries, a percentage of patients, which is not insignificant, reported reaching haemoglobin levels between $5 \mathrm{~g} / \mathrm{dL}-7 \mathrm{~g} / \mathrm{dL}$ while some reported reaching levels below 5 $\mathrm{g} / \mathrm{dL}$ and many have reported receiving whole blood. Patients from the Western countries reported milder to no pre 
transfusion haemoglobin drops, despite the observed and reported initial blood shortage. In a few countries mainly Cyprus, Greece and Italy with very strong blood donation infrastructures, historically related to the care of thalassaemia patients, the reported decrease of blood supplies was indeed a very temporary phenomenon having reverted already (by early May) almost to the pre-COVID-19 practices and supplies.

In terms of numbers, $100 \%$ of patients (survey 2) responded to the question on blood shortages as follows:

- $8 \%$ no interruptions* (100\% from Western countries)

- $38 \%$ moderate interruptions* $(78.6 \%$ from Western countries / 21.4\% from developing countries)

- $\quad 54 \%$ serious interruptions* $(100 \%$ from developing countries)

- *Interruptions in frequency and/or in quantity provided

- Restricted access of patients to their 'specialised' treatment/transfusion centres. This finding was reported almost exclusively in the developing countries with very mild discrepancies reported in the Western word which took early measures to ensure safe access of patients to their treating centres, both in terms of transport and treatment space. The patients seeking medical care and transfusion in their nearest medical/hospital settings in the developing countries, consequent to prevention/isolation measures including inaccessible public transportation, are exposed to significant risks. These are associated with the potentially confined knowledge and expertise of healthcare professionals in such settings including provision of whole blood. This inevitably may result to the development of adverse reactions and medical complications of various levels of severity;

In terms of numbers, $100 \%$ of patients (survey 2) responded to the question on safety of blood and concerns regarding blood transfusions as follows:

- $\quad 2.5 \%$ no challenges* identified $(100 \%$ from Western countries)

- $\quad 41.6 \%$ moderate challenges* identified (35.4\% from Western countries/ 64.6\% from developing countries)

- $55.9 \%$ serious challenges* identified (100\% from developing countries)

- *hallenges: (i) not well matched blood, (ii) and/or not filtered, (iii) and/or whole blood provided

- Limited to no access of patients to chelation therapy drugs and other medicines essential for their underlying disease and existing co-morbidities.
This again was/is happening mainly in the developing world where patients need to access specific nationally assigned pharmacies for these drugs which however are now closed down (or do not provide services to patients with haemoglobin disorders as their role now is to address COVID-19 needs). In addition, drug deficiencies are reported due to national restrictions of importing (innovative) and/or of locally manufacturing them (e.g. generic ones).

In terms of numbers, $100 \%$ of patients (survey 2) responded to the question on iron chelation as follows:

- $\quad 7.6 \%$ no challenges* identified $(82.5 \%$ from Western countries)

- $\quad 39.8 \%$ moderate challenges* identified (26\% from Western countries/74\% from developing countries)

- $\quad 52.6 \%$ serious challenges* identified (100\% from developing countries)

*Challenges: (i) interruption of chelation,

(ii) moderate shortages, (iii) serious shortages/no availability

Furthermore, the closing down or sub functioning of many $N G O s$, consequent to COVID-19, in many countries of the developing world which were authorised for years in the past to distribute to patients' drugs, particularly iron chelation ones, adds significantly, to the level of inaccessibility of patients to their life saving medicines.

Such findings are not reported and are almost absent in Western countries where health care systems are largely based on universal coverage with very well structured public health policies and services on chronic and genetic diseases. Particularly, organised services for haemoglobin disorders are in place in Southern European countries, Cyprus, Italy, Greece but also, UK, France and Germany which historically host immigrants from countries with high prevalence in these disorders.

Both in Western and developing countries significant social challenges occurred, including loss of jobs, with more severe consequences however for patients in the developing world who have to cope with their basic care and everyday basic living costs.

The social measures taken across the world may alleviate the situation of many patients particularly of the West. In the developing world however, no measures, irrespective of their extent, can address the huge social challenges that have occurred to patients, consequent to COVID-19; particularly taking into account the low HDI of the majority of the countries where haemoglobin disorders are most prevalent.

In terms of numbers, $100 \%$ of patients (survey 2) responded to the question on fac- ing any economic challenges as follows: $6.8 \%$ temporary challenges* $(98.6 \%$ from the Western countries)

$33.2 \%$ moderate challenges*

$60 \%$ severe challenges*

*Challenges: (i) loss of job, (ii) and/or loss of other income, (iii) and/or coping with daily expenses, (iv) and/or coping with costs of medical care

\section{Discussion and Conclusions}

Governments, health care professionals and patient communities at national and international level, should remain committed to support WHO to achieve the attainment of the United Nations Sustainable Development Goals (SDGs) 2030 including amongst others:

- Goal 1: No Poverty;

- Goal 3: Ensuring healthy lives and promoting the well-being for all at all ages is essential to sustainable development; Goal 10: Reducing inequalities;

Goal 17: Building partnerships for achieving the Sustainable Development goals

In the context of Goal 3, transition of health care systems and achievement of universal health coverage constitutes a major component and is particularly relevant to our patient population in the developing world.

In addition, other resolutions and/or recommendations and/or programmes of WHO including blood safety/adequacy, patient safety, Non Communicable Diseases (NCDs), congenital birth defects, essential medicine list, access to innovative drugs and therapies, already constitute, or will soon constitute, important focus of every Government in the context of their efforts towards improving the quality of services provided in their country and reducing existing huge inequalities in the area of health and chronic diseases.

Work has to be continued certainly in the Western world as well, particularly in the area of public health, access to innovative drugs and therapies and in addressing more effectively the heavily increasing, in current years, migrants' social and health challenges.

The concern of TIF of course now is, and quite unknown for how long in the future will be, to support with specific and targeted actions the patient community it represents across the world.

As precautionary measures, provided mainly by the WHO (since the declaration of COVID-19 as a pandemic) for the control of the spread of SARS-CoV-2, were the same for every country worldwide, the 
degree of effectiveness demonstrated by each country relied on: i) The political commitment of each Government to combat COVID019 putting at the front the public health benefit of its country's citizens irrespective of any financial consequences; ii) The promptness of each Government to adopt and implement WHO's recommended measures and the prompt development of a COVID-19 specific strategy; iii) The promptness of each Government to seek and adhere to the guidance of national and international experts and the establishment of a strict monitoring system for the implementation of all recommendations; iv) The robustness of the country's health care system in terms of primary and public health infrastructures, public hospital competence and capacities including importantly emergency and intensive care units' services.

Other factors related to the country's characteristics and environment certainly constitute important parameters to consider including:

- Country's and/or population size

- Financial resources -HDI level of the country

- Political situation

- Religious/cultural customs

and in the interest of TIF

- National programmes for chronic patients and more particularly national disease specific prevention and management programmes including registries and the

- Prevalence of patients with haemoglobin disorders

Based on the above considerations, some countries mainly in the EU (including Cyprus, Italy, Greece and France) and a few outside including Canada, Singapore, North Korea, People's Republic of China (where it all started), including Hong Kong and Taiwan, have reacted and acted promptly. Having all the favourable prerequisites, as mentioned previously, they demonstrated effectiveness in terms of reduction of infected cases, deaths and appropriate caring of hospitalised patients. USA and UK reacted with some delay in taking standard precautionary measures as those recommended by the WHO and other official bodies, and had, as a result, significantly higher (particularly in the USA) number of affected cases, deaths and needs for hospitalisation despite their robust heath care infrastructures

In all of the countries mentioned above however, the patients with haemoglobin disorders were, for many years now, almost all under optimal or nearly optimal medical care, and were, contrary to the rest of the community as seen in some of the countries mentioned above (USA and UK for example), were provided quite promptly with rel- evant recommendations, and advice by experts and every effort was made to ensure that their standard medical care remained as uninterrupted as possible during this crisis. As in all of the above mentioned countries, robust registries for haemoglobin disorders exist, the effectiveness of the country's actions could be quite reliably assessed and reported. In Cyprus, Greece, Italy, UK and France for example which published results, between 0-3 cases with thalassaemia have been reported with mild symptomatology and very quick (2-4 days) recovery and most with very brief or no hospitalisation at all. Although the number of patients with SCD as reported from these countries was higher, with more severe clinical outcomes and higher mortality rates, one must not underestimate: i) the pathology of this disease which makes it by definition more vulnerable to respiratory infections; ii) the significantly higher numbers as compared to thalassaemia in almost all of the above countries (except Cyprus); and iii) the socio-economic environment of the majority of these patients.

Published information regarding haemoglobin disorders from certain EU countries including Bulgaria and Romania and other extended European countries including Turkey, is not to date available despite the existence of national disease specific programmes including registries for haemoglobin disorders in these countries.

The reports reaching TIF from its members in the Eastern Mediterranean Region (EMRO) countries, mentioned to date, very few cases of patients with haemoglobin disorders infected with SARS-CoV-2. These countries, despite their significant progress in the area of the control and management of haemoglobin disorders through the years, have not todate published any results in relation to COVID-19. And although reports reaching TIF mention very impressively very low to zero number of affected cases, this has yet to be confirmed in the very near future. This will be plausible because many of the countries in this region have very comprehensive disease specific programmes and registries, including: Lebanon, Iraq, Iran, UAE, Bahrain, Oman, Qatar, KSA, and to a great extent Egypt, Morocco and Tunisia. One must of course underscore the huge challenges involved in compiling and accurately reporting data in a big and highly densely populated country such as Egypt and the serious political upheaval with its tragic consequences that exist in two countries of the region including Yemen and Syria and which have resulted to large population movements (refugees, migrants) with completely unknown data or information regarding the COVID-19 status.

In South-East Asia Region (SEARO), TIF's collaborators and members in India, Maldives, Indonesia and Thailand, though have not todate published any data, report to TIF nearly zero affected cases/deaths. These highly populated countries (except Maldives) are all with high prevalence of haemoglobin disorders (mainly thalassaemia) and despite their huge efforts and focus on these diseases, for some years now, the absence (with the exception of Maldives, Indonesia and to a certain extend Thailand) of nationally coordinated comprehensive disease specific strategy including registries, makes accurate and reliable diagnosis and impact of COVID-19 on patients with haemoglobin disorders indeed very challenging.

India as a subcontinent, focused considerable effort on supporting patients with haemoglobin disorders during COVID-19 and the contribution of patients and patients' organisations in this effort has been truly exemplary.

In West Pacific Region (WPRO) Philippines with considerable long-term interest in the care of thalassaemia and Malaysia of good national registry and with excellent records for its work in the control and management of haemoglobin disorders for many years now, have also not todate reported any infected cases/deaths (in patients with thalassaemia).

One must not avoid to mention the complete absence of information regarding the fate of patients with haemoglobin disorders, mainly thalassaemia, in countries of low to very low HDI level, and very high prevalence of these disorders including Vietnam, Cambodia, Nepal and Bangladesh. The situation prior to COVID-19 pandemic with regards to appropriate prevention and medical care of patients with thalassaemia was already very challenging to tragic. It will be almost impossible to trace or even grossly assess the consequences of this crisis which TIF believes are huge. The impact on the health and lives of the patients and their families is immense.

TIF believes that all of the countries outside mainly the Western sphere of the world, are still going through or just going out of the active phases of the infection and as such they need more time to assume the infection plateau stage after which they can more reliably assess the situation and extract conclusions on the impact of COVID-19 also relevant to the patients with haemoglobin disorders. It is almost certain that considerable underreporting or no reporting of symptomatic patients and/or deaths (consequent to recovering or dying 
at home with no hospitalization) exists todate. In addition, asymptomatic cases are not acknowledged since there are no testing (SARS-CoV-2-RNA) policies in place. Misdiagnosis, is also a possible phenomenon because of the widely varied clinical manifestation of this infection.

Many and multiple medical complications are reported in literature consequent to infection with SARS-CoV-2. It has been reported for example that up to $30 \%$ of patients who are seriously ill with this virus are developing thrombosis complications. From autopsy data, it has been shown that this virus has an organotropism beyond the respiratory tract including the kidneys, liver, heart and brain. It is speculated that this organotropism influences the course of COVID-19 disease and possibly aggravates pre-existing conditions.

It has also been reported to cause diarrhoea and other gastrointestinal symptoms and evidence of infectious virus has been reported in the stool of patients with the possibility of the faecal-oral route transmission of this virus raising even bigger concerns with regards to the living and hygiene standards practiced in a large proportion of our patients' population in the developing world.

TIF believes that today's information regarding the level of infection amongst patients with haemoglobin disorders, as reported to TIF and in the absence of any, relevant to haemoglobin disorders, published data from countries across the world, is not in agreement with the global official reports on the infection and morbidity rates of COVID-19 in the community at large, This is particularly impressive, as one might expect significantly greater numbers of infected patients with haemoglobin disorders than those of the general community, acknowledging the fact that: i) the greatest majority of the patients in some of these regions of the world, consequent to lifelong receipt of suboptimal or no care, have many and multiple co-morbidities; and ii) considering some emerging information (yet to be confined) of some ethnicity related higher vulnerability with regards to Asian populations.

Whatever the truth about the fate of our patients in the different parts of the world and which will hopefully be better revealed in the coming months, the reality is that the COVID-19 pandemic has brought about immense challenges in the health and social care systems and economies in every country of the world and more severe ones in countries of the developing world in which the greatest majority of our patients live.

The hope and optimism lies in the development of effective and safe vaccines and use of safe and effective therapeutic medicines. Our hope lies on the intensive research activity ongoing today in many countries across the world and on the establishment of a truly global collaboration as WHO continuously and passionately advocates in the past few months. It is essential to identify funding for the research and production of vaccine and ways to make these, when authorised and available on the market accessible to all the people and not only the privileged few. This can only happen with global coordination, collaboration and solidarity and patients with haemoglobin disorders should not be left behind but on the contrary they should be amongst the priority groups of patients.

TIF will continue to collaborate closely with its patients, medical/scientific collaborators, the $\mathrm{WHO}$, the EU, the ASEAN and other official health related bodies at national, regional and international level and will continue to strengthen its activities around the world. First and above all TIF is fighting to ensure continuity of the basic medical and other care at country level and towards further improvements which could happen mainly through universal covered heath care systems with strong primary and public health infrastructure and disease specific national programmes.

Indeed, health care systems in most developing countries, are in transition stages aiming for significant improvements. We hope that this pandemic will not contribute to the weakening of these efforts.

\section{References}

1. Williams TN, Weatherall DJ. World distribution, population genetics, and health burden of the hemoglobinopathies. Cold Spring Harb Perspect Med 2012;2:a011692.

2. Cappellini MD, Cohen A, Porter J, et al. Guidelines for the management of transfusion dependent thalassaemia [TDT]. 3rd edition Thalassaemia International Federation 2014; TIF publication 20.

3. Taher A, Musallam K, Cappellini MD. Guidelines for the management of nontransfusion dependent thalassaemia [NTDT]. 2nd edition. Thalassaemia international Federation 2017; TIF publication 22.

4. Farmakis D, Giakoumis A, Angastiniotis M, Eleftheriou A. The changing epidemiology of the ageing thalassaemia populations: A position statement of the Thalassaemia International Federation. Eur J Haematol 2020;10.1111/ejh.13410. doi:10.1111/ejh.13410 [Epub ahead of print]. 